\title{
Electrical Probing of Silver Nanowires in situ Transmission Electron Microscopy
}

\author{
Diego Alducin ${ }^{1}$, John E. Sanchez ${ }^{1}$, Miguel José-Yacaman ${ }^{1}$, and Arturo Ponce ${ }^{1}$ \\ 1. University of Texas at San Antonio, Department of Physics and Astronomy, San Antonio, TX, USA
}

One of the most important aspects of the area of material sciences is the characterization of nanostructured and advanced materials. Silver is one of the most promising materials due to the large thermal and electrical conductivity compared to other metals. However, not all properties of materials scale down in proportion to its one-dimensional nanostructure. In this work we present the electrical behavior of single silver nanowires. By employing an in situ transmission electrical holder, one can measure the resistivity and conductance of individual nanowires while observing the changes of the structure due to the high resolution transmission electron microscope. By applying different voltages we observed the how the nanowire is affected by this increase until failure.

Using the polyol method we obtained uniform silver nanowires with a high yield. Figure 1 (a) shows a scanning electron microscopy image of purified Ag NWs. The average diameter of the nanowires was $\sim 60 \mathrm{~nm}$ and the length varied from 10 to 20 micrometers with the distribution as the inset of Figure 1 (a). Figure 1 (b) shows a magnified SEM image in which it is noted the pentagonal section of one nanowire, the (100) and (111) facets are shown as a simulation in the inset image. In Figure 1 (c) the end of an individual Ag NW is presented. The nanowire growth is along <110> direction. From the high magnification micrograph shown in Figure $1(\mathrm{~d})$, the measured lattice distances are $0.28 \mathrm{~nm}$ and $0.25 \mathrm{~nm}$ corresponding to the $\{110\}$ and $\{111\}$ planes of the cubic structure of silver, respectively. In this study, the mechanical properties of the nanowires were tested until fracture in a three point test [2].

Electrical measurements where performed using a dedicated Nanofactory Instruments AB holder. Silver nanowires are deposited to a half a copper grid which is connected to the instrument controller. A tungsten probe is controlled by a piezoelectric transducer that can be manipulated in fine movements of up to $1 \mathrm{~nm}$ in step size. The probe can approach the silver nanowire and touch the protruding end of the structure and complete the conductance measurements. The measurements were observed and recorded using a transmission electron microscope JEOL 2010F with an ultra-high quality CMOS camera attachment.

Previous work has shown how the electrical properties of polytypic nanowires [1] however we provide the difference in electrical conductance due to the fracture of the wire. This work also shows how the nanowire fails under high voltages. Figure 2 (a) (b) shows the I-V curve and dark field image of the silver nanowire without fracture during measurement. Figure 2 (c) (d) shows the current - voltage curve of the silver nanowire after a fracture is introduced into the structure. Our results show that the conductance of the nanowire is affected by the changes in the structure, specifically by fracture. [3]

\section{References:}

[1] X Liu et al, Nanotechnology 19 (2008), 085711

[2] D Alducin et al, Scripta Materialia 113 (2016) 63-67

[3] The authors acknowledge funding from the Welch Foundation (AX-1615), the Department of Defense \#64756-RT-REP, the National Insitute on Minority and Health Disparities (NIMHD) in the program Research Centers in Minority Institutions Programs (RCMI) Nanotechnology and Human 
Health Core (G12MD007591), and the Mexican Council for Science and Technology, CONACYT \#250836 (Mexico).
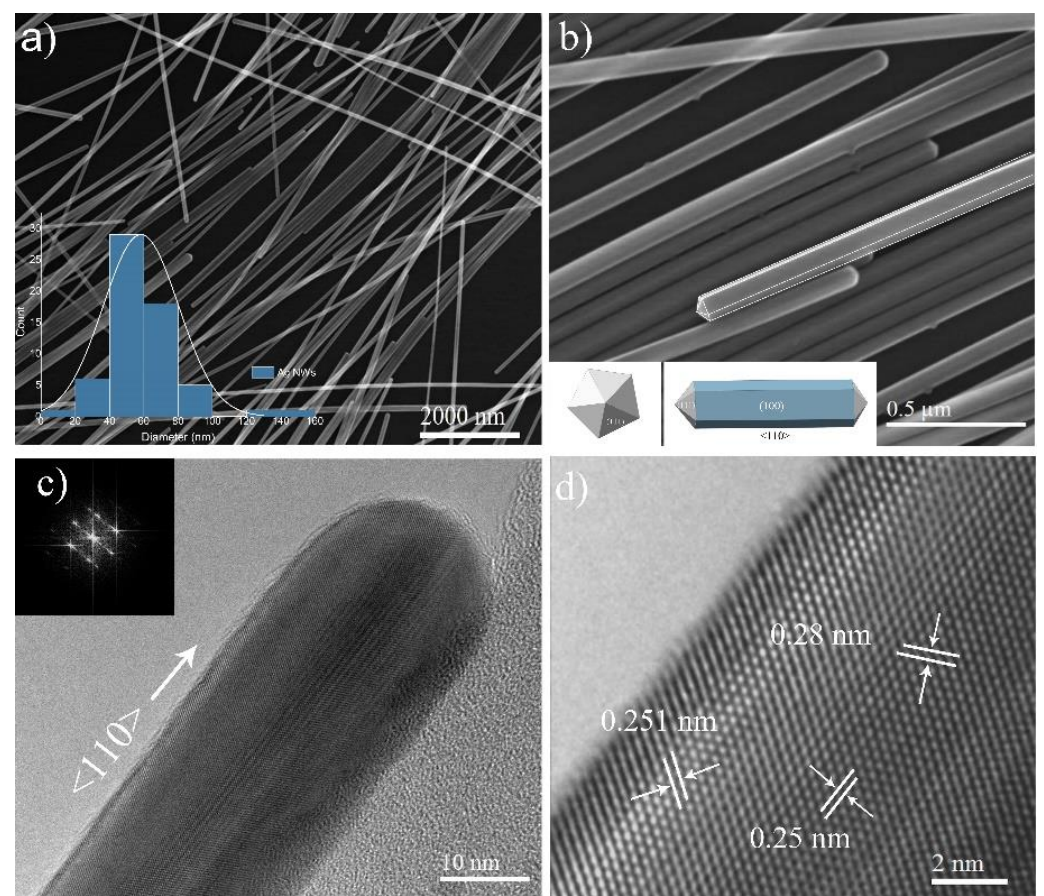

Figure 1. Micrographs of the synthesized Ag nanowires. (a) Low magnification SEM image nanowires. (b) High magnification SEM image in which the pentagonal cross section of one nanowire is observed. (c) TEM micrograph of the tip of one nanowire. The FFT shows the characteristic reflections of a twinned structure. (d) HRTEM image showing the lattice fringes of the nanowire.
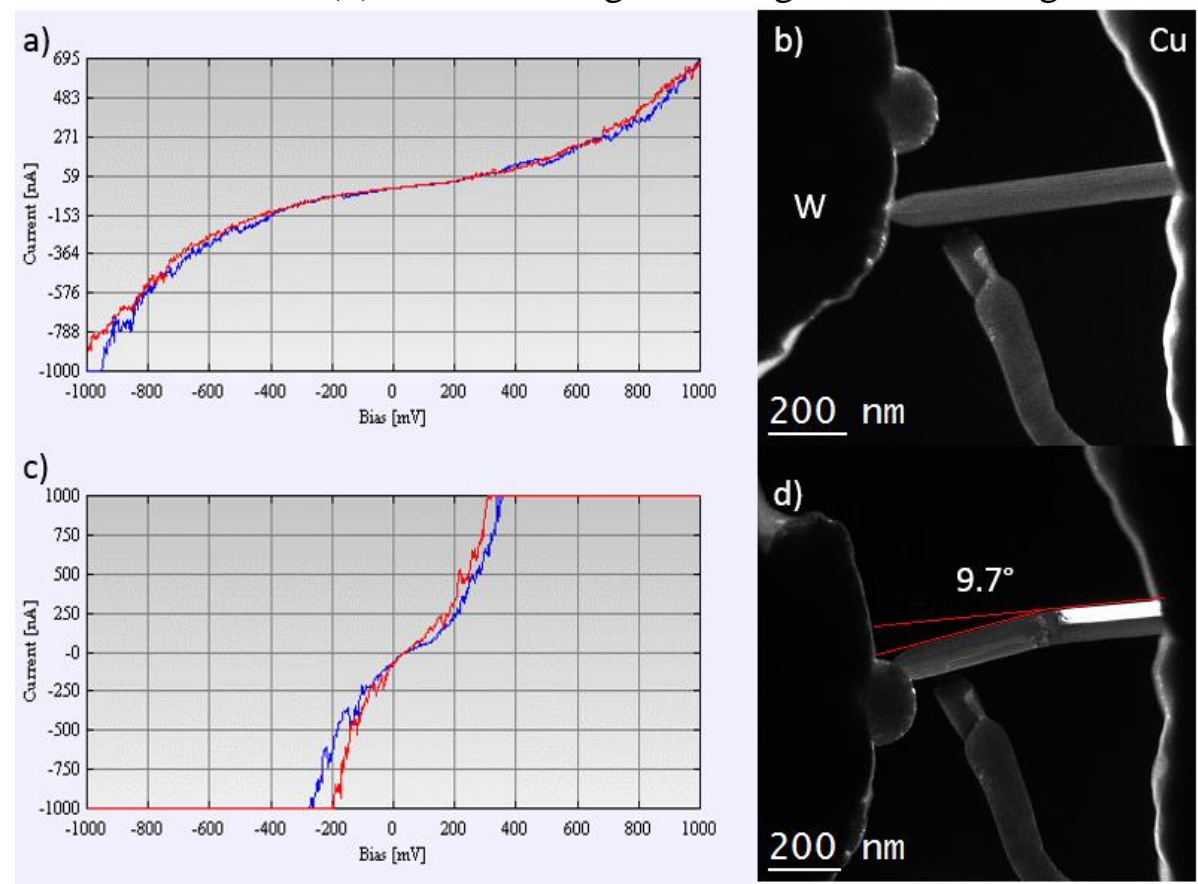

Figure 2. a) Current - Bias curve of the complete nanowire. b) TEM dark field image of the nanowire during the measurement. c) Current - Bias curve of the nanowire with a fracture at an angle $9.7^{\circ}$ from the original position. d) TEM dark field image of the fractured nanowire during the measurement. 